\title{
Medios electrónicos y estado nutricional antropométrico en escolares
}

\section{Electronic media and anthropometric nutritional status in schools}

DOI: http://dx.doi.org/10.17981/cultedusoc.12.1.2021.14

Recibido: 4 de mayo de 2020 Aceptado: 17 de julio de 2020 Publicado: 28 de diciembre de 2020

\author{
Hendrik Rafael Márquez-Luengo (D) \\ Universidad del Zulia. Maracaibo (Venezuela) \\ hmarquezl39@gmail.com
}

Darío Acosta

Cáritas de Venezuela. Maracaibo (Venezuela)

dario.dajh@gmail.com

\author{
Jorge Luis Chourio \\ Fe y Alegría. Maracaibo (Venezuela) \\ jorgelchch@gmail.com
}

José Reverón (1)

Mcg consultores. Santiago (Chile)

josreveron@gmail.com

Para citar este artículo:

Márquez-Luengo, H., Acosta, D., Chourio, J. y Reverón, J. (2021). Medios electrónicos y estado nutricional antropométrico en escolares. Cultura, Educación y Sociedad, 12(1), 217-226. DOI: http://dx.doi.org/10.17981/cultedusoc.12.1.2021.14

\section{Resumen}

A partir de la primera infancia pueden surgir modificaciones en las conductas y formas comportamentales, es decir, de actividades recreativas intensas a actividades mínimas o ligeras como mirar equipos o medios electrónicos, así como dispositivos móviles. Desde esta premisa, el artículo se orienta a evaluar el uso de medios electrónicos y el estado nutricional antropométrico en estudiantes en edad escolar. Se realizó una investigación descriptiva, transeccional y de campo, a una población de 120 escolares entre 7 y 12 años de un colegio privado de la ciudad de Maracaibo (Zulia, Venezuela); los cuales recibieron evaluación antropométrica, y se les aplicó un cuestionario estructurado para conocer sus percepciones en torno al uso de los medios electrónicos. La información fue procesada mediante estadística descriptiva. Los resultados evidencian en los niños valores que describen un estado antropométrico normal; así mismo, se identifican como medios electrónicos más utilizados el televisor y el computador. Se concluye en la importancia de reconocer la idea de que el entorno digital y la dependencia de equipos electrónicos forman parte de la vida y la cotidianidad de los niños.

Palabras clave: Escolares; medios electrónicos; estado nutricional; salud integral

\section{Abstract}

From early childhood, modifications in behaviors and behavioral forms can arise, that is, from intense recreational activities to minimal or light activities such as looking at electronic equipment or media, as well as mobile devices. From this premise, the article aims to evaluate the use of electronic media and the anthropometric nutritional status in school-age students. A descriptive, transectional and field investigation was carried out on a population of 120 schoolchildren between 7 and 12 years old from a private school in the city of Maracaibo (Zulia, Venezuela); who received an anthropometric evaluation, and a structured questionnaire was applied to know their perceptions about the use of electronic media. The information was processed using descriptive statistics. The results show in the children values that describe a normal anthropometric state; Likewise, the television and the computer are identified as the most used electronic means. It concludes on the importance of recognizing the idea that the digital environment and dependence on electronic equipment are part of the lives and daily lives of children.

Keywords: Schoolchildren; electronic media; nutritional status; integral health 


\section{INTRODUCCIÓN}

La edad escolar constituye un período decisivo para la modificación de conductas alimentarias y otros estilos de vida que perdurarán en todas las etapas con repercusiones no solo en la niñez, sino también en la edad adulta. Camargo y Orozco (2013) señalan que "es la etapa de la vida en que avivan su desarrollo intelectual, consolidan sus capacidades físicas, aprenden los modos de relacionarse con los demás y aceleran la formación de su identidad y su autoestima" (p. 176). Un adecuado uso de dispositivos móviles y equipos electrónicos ofrece al estudiante de educación básica alternativas de índole académico; pero un inadecuado uso puede interferir en el rendimiento académico y a su vez sobre las horas de sueño permitidas (Cerisola, 2017).

Ahora bien en los estudiantes, el uso de medios electrónicos de esparcimiento, tal es el caso del televisor, equipos que garanticen o no desplazamientos, laptop y equipos que necesiten de conexión, son parte de los hábitos de los escolares. Cabe destacar que la utilización de estos equipos beneficia el esparcimiento y al veloz acercamiento a los mensajes comunicacionales (Chávez, 2014).

Sin embargo, el uso abusivo de medios electrónicos y de esparcimiento puede ocurrir que los escolares descuidan la hora adecuada de su alimentación, con ello afectan su estado nutricional antropométrico, producto de una disminución en la ingesta diaria, y a su vez puede traer problemas a nivel del aparato digestivo superior e inferior.

De acuerdo con lo anterior, Camargo y Orozco (2013) señalan que "desde la primera infancia pueden surgir modificaciones en las conductas, es decir, de actividades recreativas intensas a actividades mínimas o ligeras como mirar equipos o medios electrónicos, así como dispositivos móviles" (p. 176).

Los mencionados autores resaltan además que:

El uso de estos medios se debe a causas como abandono del hogar por parte de los padres, disminución en la seguridad de las ciudades; igualmente se ubican los diseños curriculares planificados por docentes, donde hacen énfasis en actividades más pedagógicas que actividades orientadas a aptitudes deportivas y de creación de los estudiantes. A parte del acceso que tienen a equipos electrónicos en el hogar iniciando a temprana edad (Camargo y Orozco, 2013, p. 176).

Incluso en el ámbito escolar la integración de medios electrónicos a los procesos didácticos puede representar un agente inhibidor o dinamizador de la relación entre homólogos, es decir los procesos convivenciales pueden afectarse por factores endógenos o exógenos. Al respecto, Mena y Huneeus (2017) asocian la convivencia en al escuela desde el fortalecimiento de las relaciones entre los actores educativos.

Por otro lado, Ruiz (2015) mencionan que ante la situación planteada, el uso oportuno o no de las tecnologías, están ocasionando mayores niveles de sedentarismo en escolares lo cual denota preocupación a nivel global, esto ha hecho que los organismos internacionales hayan elaborado recomendaciones especiales para velar por la salud de estos. En este sentido, la recomendación principal es limitar el uso de programas multimedia máximo a $1-2$ horas diarias, y que estas sean retiradas de sus alcobas. Dado que exceder el tiempo mirando pantallas puede dificultar el cumplimiento de otras labores interactivas que resultan primordiales para un proceso natural en la niñez y adolescencia. 
Silva (2014) menciona que un "tercio de los niños del planeta se pasan al menos tres horas al día frente al televisor o del computador" (p. 3), según un informe anual de la Organización Mundial de la Salud (OMS) en el 2007, realizado a 70.000 niños de 34 países. La misma OMS (2016) describió cifras alarmantes de 340 millones de niños, niñas y adolescentes (de 5 a 19 años) con malnutrición por exceso, más de 1900 millones de adultos de 18 o más años con Índice de Masa Corporal (IMC) entre 24.9\% 29.9\% y 650 millones con IMC superior a 30\%. En este sentido, las estadísticas de malnutrición por exceso han aumentado a nivel global considerándose actualmente como un asunto público.

En este orden de ideas, la malnutrición por exceso en niños y niñas tiene un impacto sobre la salud y el estilo de vida tanto a corto como a largo tiempo. Entre el impacto se ha señalado en reportes e informes, que niños y adolescentes con problemas de malnutrición por exceso tienen más riesgo para contraer enfermedades crónicas no transmisibles (cardiovasculares) como colesterol y triacilglicéridos elevados, hipertensión arterial, también mayor riesgo de padecer diabetes mellitus, problemas óseos y articulares, respiratorios a la hora de dormir, así como también problemas en lo social y colectivo, psicológicos como la estigmatización y baja autoestima (Solis, 2014).

Cabe destacar que el sedentarismo en este grupo de edad es relevante. Según Rojo (2015), hallazgos de la Encuesta Nacional de Salud de España realizada en los años 2011/12 señalan que 04 de cada 10 sujetos adultos jóvenes manifiestan ser sedentarios en su período de descanso. Así mismo el 44.4\% de la muestra estudiada de 15 y más años declaran que no realiza actividades físicas y que ocupa su período de descanso de manera totalmente sedentaria. Con respecto al grupo menores de 15 años, el $12.1 \%$ de los niños manifiestan ser sedentarios, es decir, no realizan ejercicios y deporte alguno en su tiempo libre.

En este sentido, Domínguez-Anaya, Echávez y Tapia (2014) exponen:

[...] que los estudiantes seleccionan aquellas labores donde no aplican movimiento de ninguna índole y prefieren actividades dedicadas a la utilización de computadoras y televisor, el cálculo de este hábito creado ofrece una perspectiva de todos los estadios de sedentarismo; por esta razón la televisión es valorada por países occidentales como la causa principal de inacción, a medida que aumente el uso de este equipo hay mucha probabilidad que se relacione el aumento de la malnutrición por exceso (p, 34).

Dadas las condiciones que anteceden, la ausencia de actividades recreativas físicas aumentan las probabilidades de padecer malnutrición por exceso, y en algunos casos, el afán a la utilización de estos medios es tan excesivo que olvidan comer para nutrirse mejor. Domínguez et al. (2014) mencionan que debe haber una "armonía en las horas de descanso dedicadas a la labor corporal y la utilización de medios es primordial para una excelente condición nutricional, sugieren que la actividad física, ejercicios, el deporte y el sedentarismo están relacionados directamente con la condición nutricional" (p. 34). La intensidad en la inactividad física es elevada en varios países (OMS, 2015), todo esto, motivado al avance exhaustivo de modernas tecnologías que se apropian del espacio de descanso de las personas, el traslado a sitio de trabajo, entre otras.

Por las consideraciones anteriores, este artículo tiene como objetivo evaluar el uso de los medios electrónicos y el estado nutricional antropométrico en escolares de educación básica en la ciudad de Maracaibo (Venezuela); de esta manera se trabaja como variable interviniente en la situación problema, el uso prolongado por parte de la muestra seleccionada de los medios tecnológicos identificados. 


\section{Metodología}

El estudio fue descriptivo, transversal y de campo; y la población se conformó con la totalidad de estudiantes pertenecientes a todos los grados y secciones de una institución educativa de la ciudad de Maracaibo (Zulia, Venezuela). La muestra estuvo representada por sujetos de ambos sexos de educación básica, con edad comprendida entre 7 y 12 años, siendo incluidos solo aquellos que cumplían con los requisitos establecidos, dicha cifra se obtuvo por medio del cálculo de la muestra. Se aplicó la fórmula de Sierra (1994) para calcular la muestra, el resultado fue: 117. Es decir, que, de la población de 465 estudiantes de educación básica, se seleccionaron 120 estudiantes que se organizaron según estratos (Hernández, Fernández y Baptiste, 2006). La distribución de la muestra quedó conformada por los estudiantes de Educación básica de los diferentes grados; el proceso se desarrolló de manera aleatoria evaluándose 20 escolares de cada grado y sección para un total de 120 escolares. Vale destacar que los Escolares contaron con la aprobación de sus padres para tomar parte del estudio.

La técnica empleada fue la entrevista de tipo personal, utilizando para el logro de la información un guión de entrevista, la cual permite, recabar o registrar toda la información relacionada con la variable de evaluación nutricional y preguntas relacionadas sobre el uso de medios electrónicos. Con respecto al estado nutricional antropométrico se obtuvo a través de las valoraciones antropométricas. En la toma de los datos antropométricos de los escolares, el peso se determinó en una balanza bioeléctrica TANITA (TBF 300 GS-TBF MA), que expresó la medición en kilogramos con graduaciones mínimas de 100 gramos y con capacidad máxima de 150 kilogramos. Para iniciar esta medición se aseguró que la balanza se encuentre sobre una superficie horizontal y plana, el sujeto sin los zapatos y ropa ligera, luego se coloca al centro de la balanza, en posición de firme, relajado, con los miembros superiores pegados a ambos lados del cuerpo, la vista al frente y el peso del cuerpo distribuido entre ambos pies. Se registró el número (peso) del sujeto en kilogramos (Nariño, 2016).

La medición de la talla se realizó con una cinta métrica convencional de $150 \mathrm{~cm}$, se colocó la cinta métrica en una pared lisa, con piso nivelado y se fijó en la pared con cinta adhesiva y ayuda de una plomada para asegurar que se fijase en una línea recta paralela a la plomada. Posteriormente se procedió a medir a cada uno de los individuos, tomando en cuenta las siguientes características: sin zapatos y en posición erecta, con los pies unidos; talones, nalgas, espalda y región occipital pegados con el plano vertical de la cinta métrica. La medición se realiza con los individuos mirando hacia el frente (Geraldo, 2015). Finalmente se colocó una escuadra en la parte superior de la cabeza y se procedió a la lectura de la medida en centímetros.

El diagnóstico nutricional antropométrico se obtuvo mediante la combinación de los indicadores P/E, P/T, T/E, CMB y CC y de la obtención del Índice de Masa Corporal (IMC). Los indicadores resultaron al graficar la talla, edad y peso, a través de las gráficas de la OMS modificadas por el instituto operacional de la nación en materia de Nutrición el Instituto Nacional de Nutrición (INN), con un rango de normalidad en los percentiles 10 y 90 (un rango por encima del percentil 90 indica exceso y por debajo del percentil 10 déficit). 
Para la descripción de los datos de esta investigación, se realizaron cálculos mediante estadísticas descriptivas, reportando los valores de frecuencia absoluta, frecuencia relativa y promedio para cada una variable. Todos los datos del estudio se analizaron con el software SPSS IBM (versión 22.0). A las variables objeto de estudio se les aplicó un cuestionario contentivo de aseveraciones, organizadas en respuestas abiertas. Para la construcción de los instrumentos se tomó en cuenta la matriz de operacionalización de las variables, considerando una dimensión nominal, operacional, subdimensiones e indicadores asociados.

A dichos instrumentos se le aplicó las pruebas de validez de contenido por medio del juicio de expertos quienes evaluaron la pertinencia, redacción y coherencia de cada uno de los reactivos en correspondencia con el contexto teórico y empírico, así como también su relación con los objetivos de investigación.

\section{Resultados}

En el presente apartado se visualizan los resultados reportados por el análisis estadístico, haciendo referencia a las variables medidas en el uso de los medios electrónicos y estado nutricional antropométrico en escolares. Específicamente, se hace uso de tablas de distribución de frecuencias absolutas y relativas. En la Tabla 1 se observa que la población estudiada estuvo conformada por 120 sujetos de los cuales 66 eran femeninos y 54 masculinos.

TABLA 1.

Distribución de la población por sexo de los estudiantes evaluados

\begin{tabular}{llll}
\hline \multicolumn{1}{c}{ Género } & & $(\mathrm{n})$ & $(\%)$ \\
\hline Masculino & 54 & & 45 \\
Femenino & 66 & 55 & \\
Total & 120 & 100 \\
\hline
\end{tabular}

Fuente: Márquez, Acosta, Chourio y Reveron (2020).

La Tabla 2 señala el diagnóstico nutricional antropométrico de la muestra seleccionada observando que hubo un predominio de la normalidad con respecto al estado nutricional con un $66.6 \%$ siguiendo el sobrepeso con un $15 \%$ y en menor proporción la obesidad con un resultado de $4.16 \%$.

TABLA 2.

Diagnóstico antropométrico de los escolares

\begin{tabular}{|c|c|c|}
\hline Diagnostico antropométrico & (n) & $(\%)$ \\
\hline Déficit & 17 & 14.16 \\
\hline Normal & 80 & 66.66 \\
\hline Sobrepeso & 18 & 15 \\
\hline Obesidad & 05 & 4.16 \\
\hline Total & 120 & 100 \\
\hline
\end{tabular}

Fuente: Márquez et al. (2020). 
En la Tabla 3 se evalúan los equipos electrónicos con los que cuenta la muestra de estudio, y se puede observar que la población dispone mayormente de televisor en un 100\%, computador en un $80.83 \%$, luego la tablet con un $73.3 \%$ y en menor proporción los videos juegos con un $58.33 \%$.

TABLA 3.

Equipos electrónicos utilizados por los escolares

\begin{tabular}{llllllll}
\hline \multicolumn{1}{c}{ Tiempo de uso de equipos electrónicos } \\
\hline \multicolumn{2}{c}{ Equipos electrónicos } & \multicolumn{2}{c}{$>$ Horas } & \multicolumn{2}{c}{$<$ Horas } & \multicolumn{2}{c}{ No utilizan } \\
\cline { 2 - 8 } & \multicolumn{2}{c}{$(\%)$} & $(\mathrm{n})$ & $(\%)$ & $(\mathrm{n})$ & $(\%)$ & $(\mathrm{n})$ \\
\hline Teléfono & 48.33 & 58 & 14.16 & 17 & 37.5 & 45 \\
Computador & 51.66 & 62 & 29.16 & 35 & 19.16 & 23 \\
Tablet & 54.16 & 65 & 19.16 & 23 & 26.66 & 32 \\
Televisor & 60 & 72 & 40 & 48 & 0 & 0 \\
Video Juego & 35.83 & 43 & 22.5 & 27 & 41.66 & 50 \\
Total & 50 & 60 & 25 & 30 & 25 & 30 \\
\hline
\end{tabular}

Fuente: Márquez et al. (2020).

La Tabla 4 muestra el tiempo de uso de los medios electrónicos por parte de los escolares, arrojando que el uso "mayor de 2 horas" lo ocupan con el televisor en un 60\%; sigue el uso de la tablet con un 54\%, y en menor uso los videojuegos con un $35.8 \%$ en cambio, el uso menor de 2 horas predomina el uso del computador.

TABLA 4.

Tiempo dedicado a los medios electrónicos de los escolares del estudio

\begin{tabular}{llcccccc}
\hline \multicolumn{7}{c}{ Tiempo del uso de equipos electrónicos } \\
\hline \multirow{2}{*}{ Equipos electrónicos } & \multicolumn{2}{c}{$>2$ Horas } & \multicolumn{2}{c}{$<2$ Horas } & \multicolumn{2}{c}{ No utilizan } \\
\cline { 2 - 8 } & \multicolumn{2}{c}{$(\mathrm{n})$} & $(\%)$ & $(\mathrm{n})$ & $(\%)$ & $(\mathrm{n})$ & $(\%)$ \\
\hline Teléfono & 58 & 48.33 & 17 & 14.16 & 45 & 37.5 \\
Computador & 62 & 51.66 & 35 & 29.16 & 23 & 19.16 \\
Tablet & 65 & 54.16 & 23 & 19.16 & 32 & 26.66 \\
Televisor & 72 & 60 & 48 & 40 & - & - \\
Video Juego & 43 & 35.83 & 27 & 22.5 & 50 & 41.66 \\
\hline
\end{tabular}

Fuente: Márquez et al. (2020).

En la Tabla 5 se muestra el propósito de uso de los medios electrónicos de los infantes evaluados, arrojando que, en la población masculina el principal objetivo está relacionado al entretenimiento con un $26.66 \%$ y en la población femenina lo utiliza con diversos fines, como entretenimiento, estudio y redes sociales con un $28.33 \%$. 
TABLA 5.

Propósito de la utilización de los medios electrónicos según el sexo

\begin{tabular}{llcccccccc}
\hline \multirow{2}{*}{ Unidad de Análisis } & \multicolumn{2}{c}{ Entretenimiento } & \multicolumn{2}{c}{ Estudio } & \multicolumn{2}{c}{ Redes Sociales } & \multicolumn{2}{c}{ Todos } \\
\cline { 2 - 9 } & \multicolumn{2}{c}{$(\mathrm{N})$} & $(\%)$ & $(\mathrm{N})$ & $(\%)$ & $(\mathrm{N})$ & $(\%)$ & $(\mathrm{N})$ & $(\%)$ \\
\hline Masculino & 32 & 26.66 & 7 & 5.8 & 14 & 11.66 & 18 & 15 \\
Femenino & 32 & 26.66 & 5 & 4.1 & 19 & 15.83 & 34 & 28.33 \\
No Usa & 56 & 46.66 & 98 & 81.66 & 87 & 72.5 & 68 & 53.33 \\
\hline
\end{tabular}

Fuente: Márquez et al. (2020).

\section{DiscUsión}

En la actualidad no se evidencian estudios suficientes sobre el uso de los medios electrónicos y estado nutricional antropométrico en escolares en Venezuela, a pesar del fácil acceso que tiene la población a las tecnologías como la televisión y la computadora, por tanto, se dificulta hacer comparaciones y/o análisis entre diferentes procesos de investigación. Sin embargo, a nivel mundial varios trabajos señalan que el uso de medios electrónicos ha aumentado desde edades muy tempranas, estableciendo interrogantes del impacto que puede provocar su uso en muy temprana edad (Cerisola,2017).

Desde los primeros años de vida, los niños al crecer y desarrollarse en ambientes donde se dispone de una gran diversidad de equipos electrónicos se motivan a su uso y apropiación, aumentando así las estadísticas en la tenencia de estos equipos. Se afirma que es a partir de los 4 meses los infantes han tenido contacto con algún equipo electrónico, generalmente estos niños los denominan "nativos digitales", porque se desarrollan en ambiente donde abundan los equipos electrónicos específicamente los teléfonos inteligentes (Cerisola,2017).

Con respecto al estado nutricional antropométrico hubo un predominio de la normalidad, en concordancia con un estudio de Rodríguez (2019), cuyo resultado predominó el estado antropométrico normal. En el presente estudio los encuestados afirmaron utilizar los diferentes tipos de medios electrónico: televisor, teléfono, computadora y videos juegos, lo que indica reconocer la idea de que el entorno digital y la dependencia de equipos electrónicos son parte principal del estilo de vida de los niños hoy en día.

En este contexto, se evaluaron los equipos electrónicos con los que cuenta la muestra del estudio, se demostró que un 100\% posee televisor, seguido del computador con $80.83 \%$, y en menor proporción los videos juegos con un 58.33 \%. Estos resultados difieren con el trabajo realizado por Espinoza (2018), donde se expone que según el tipo de escuela y el factor tecnológico que más utilizan los niños (27.0\%), utilizan la televisión, el 15.3\%, las tablets con el 14.3\%, la computadora con el 12.7\%, y el teléfono móvil con el $11.6 \%$.

Con relación al uso promedio de los equipos fue de 50\% más de dos horas; se puede destacar que los datos difieren con un estudio de Chacón (2015), en lo relacionado con el uso de juego, cuyo resultado fue de 41.6\% más de 2 horas. Ahora bien, la Academia Americana de Pediatría sugiere no utilizar los equipos electrónicos más de 2 horas, ya que de 3 a 6 horas se considera abusivo y más de 6 enfermizo. Sin embargo, la utilización de estos equipos puede beneficiar al esparcimiento y a la obtención de información para actividades académicas siempre y cuando no se excedan las horas sugeridas (Chávez,2014). 
Resulta oportuno destacar que la tecnología se ha ido implementando cada vez más en los hogares, en comparación del año 2007 donde se evidenciaba un porcentaje de 55\%, para el 2015 este valor sube a 83\%, por lo tanto, los niños pasan la mayor parte de tiempo libre frente a los diferentes factores tecnológicos, como computadoras, tablets, teléfonos móviles y videojuegos (Golpe, Isorna, Gómez y Rial, 2017).

En cuanto al propósito de uso de los medio electrónicos de los escolares evaluados, los resultados concuerdan con un estudio realizado por Huamán, Palomino, Atarama y Castañeda (2016), donde señala que los escolares consideran la conexión a internet como un mecanismo obligatorio en su existencia, por esta razón ellos se unen diariamente desde su dispositivo móvil o computador para descargar espacios tecnológicos (películas, videos, series). Cabe agregar que la red más utilizada es el Facebook.

Después de las consideraciones anteriores, el rendimiento académico se puede ver afectado ya que los escolares no le asignan el tiempo suficiente para sus obligaciones educativas, pasan más horas conectados para chatear en redes inclusive en las horas de clases, complicándose su educación. Sin embargo, Zabaleta (2012) señala que "a pesar de los factores negativos y las desventajas asociados a este tipo de tecnología en edades tempranas, algunos de los dispositivos tecnológicos impulsan el desarrollo y aprendizaje de los niños pequeños utilizándolos como recurso de enseñanza y entretenimiento" (p. 4). Se puede inferir que tanto los padres como las autoridades de instituciones educativas, deben educar a los escolares sobre el uso y/o utilización de los medios electrónicos con la finalidad de emplearlos para contribuir específicamente a la educación.

\section{Conclusiones}

Se concluye en la importancia de reconocer la idea de que el entorno digital y la dependencia de equipos electrónicos forman parte de la vida y la cotidianidad de los niños. Por tanto se infiere la necesidad de que el uso adecuado de estos recursos puede representar un agente inhibidor o dinamizador del proceso de aseguramiento del aprendizaje.

Los escolares evaluados presentaron un estado nutricional antropométrico normal, lo que permite concluir que no existe un grado de afectación por parte de estos recursos sobre el desarrollo biológico de los estudiantes. Resulta pertinente analizar la relación de la variable medios electrónicos con relación a otras variables como desarrollo cognitivo y biopsicosocial de forma tal que se tenga una visión más completa y sistematizada del objeto de estudio.

La investigación destacó que los medios electrónicos representan un canal muy eficaz para el esparcimiento y recreación de los estudiantes, sin embargo, es necesario que los diferentes actores entre los que se mencionan padres y docentes puedan realizar una labor de acompañamiento y orientación, más que de fiscalización con la intención de fortalecer conductas adecuadas y formas comportamentales responsables y conscientes frente al uso de la tecnología.

\section{Agradecimientos}

Personal directivo, docente y empleados del Colegio por su disposición en la ejecución del proyecto. 


\section{REFERENCIAS}

Camargo, D. y Orozco, L. (2013). Factores asociados a la disponibilidad y uso de medios electrónicos en niños desde preescolar hasta $4^{\circ}$ grado. Biomédica, 33, 175-185. https:// doi.org/10.7705/biomedica.v33i2.779

Cerisola, A. (2017). Impacto negativo de los medios tecnológicos en el neurodesarrollo Infantil. Pediátrica de Panamá, 46(2), 126-131. Disponible en: https://pesquisa.bvsalud. org/portal/resource/pt/biblio-848347

Chacón, R. (2015). Exergames para la mejora de la salud en niños y niñas en edad escolar: estudio a partir de hábitos sedentarios e índices de obesidad. RELATEC, 14(2), 39-50. https://doi.org/10.17398/1695-288X.14.2.39

Chávez, S. (2014). Relación de las horas libres destinadas a la utilización de medios electrónicos de entretenimiento y comunicación con la actividad física y estado nutricional de los adolecentes. [Tesis pregrado]. Pontificia universidad católica del Ecuador. Quito, Ecuador. Disponible en http://repositorio.puce.edu.ec/handle/22000/7512

Domínguez-Anaya, R., Echávez, P. y Tapia, E. (2014). Diferencias del estado nutricional y hábitos de vida en escolares de 10 a 16 años de edad de una institución privada en Cartagena. Ciencia Actual, 3, 33-38. https://pdfs.semanticscholar.org/5f50/8bdd64a4 6099a671b5b4bb40aa11bfb2ae88.pdf

Espinoza, M. (2018). Factores tecnológicos que influyen en el estado nutricional de los niños de 7 a 11 años de una escuela urbana y rural del cantón chordeleg en el periodo marzo agosto del 2018. [Tesis pregrado]. Universidad Católica de Cuenca, Cuenca, Ecuador. Disponible en https://dspace.ucacue.edu.ec/handle/ucacue/6559

Geraldo, A. (2015). Ergonomía y Antropometría aplicada con criterios ergonómicos en puestos de trabajo en un grupo de trabajadoras del subsector de autopartes en Bogotá, DC, Colombia. Revista Republicana, 2-3, 135-150. Disponible en http://ojs.urepublicana.edu.co/index.php/revistarepublicana/article/view/121

Golpe, S., Isorna, M., Gómez, P. y Rial, A. (2016). Uso problemático de Internet y adolescentes: el deporte sí importa. Retos, (31), 52-57. https://doi.org/10.47197/retos.v0i31.44248

Hernández, R., Fernández, C. y Baptiste, P. (2006). Fundamentos De Metodología de la Investigación. México, D.F.: McGraw-Hill.

Huamán, F., Palomino, H., Atarama, T. y Castañeda, L. (2016). Los escolares en la era digital: el consumo de medios de los alumnos de 5to de secundaria de los colegios públicos de la región Piura en Perú.Comuni@cción, 8(1), 16-25. Disponible en https:// www.comunicacionunap.com/index.php/rev/article/view/149

Mena, M. y Huneeus, M. (2017). Convivencia Escolar para el aprendizaje y buen trato de todos: Hacia una mejor comprensión del concepto. Cultura Educación y Sociedad, 8(2), 9-20. https://doi.org/10.17981/cultedusoc.8.2.2017.01

Nariño, L. (2016). Antropometría. Análisis comparativo de las tecnologías para la captación de las dimensiones antropométricas. EIA, 13(26), 47-59. https://doi.org/10.24050/ reia.v13i26.799 
OMS. (2016). Obesidad y sobrepeso. [Online]. Recuperado de: https://www.who.int/es/newsroom/fact-sheets/detail/obesity-and-overweight

OMS. (2015). Estrategia mundial sobre régimen alimentario, actividad física y salud. [Online]. Recuperado de: https://www.who.int/dietphysicalactivity/factsheet_inactivity/es/

Rojo, M. (2015). El sedentarismo en edad escolar. promoción de un estilo de vida activo. [Tesis Pregrado]. UVA, España. Disponible en http://uvadoc.uva.es/handle/10324/14747

Rodríguez, R. (2019). Hábitos alimenticios, uso de dispositivos electrónicos y práctica de actividad física: Factores que influyen en la alteración del Índice de masa corporal en escolares de 6 a 12 años. [Tesis postgrado]. Universidad Autónoma de Aguas Calientes, Aguascalientes, México. Disponible en http://hdl.handle.net/11317/1772

Ruiz, A. (2015). Tiempo en Pantalla en Estudiantes de Cultura, Física Deporte y Recreación de la Universidad Santo Tomás, II Periodo Académico del 2014. [Tesis pregrado]. USTA, Bucaramanga, Colombia. Disponible en https://hdl.handle.net/11634/891

Sierra, R. (1994). Técnicas de Investigación Social. (7 ed.). Madrid: Paraninfo.

Silva, A. (2014). Relación de las horas libres destinadas a la utilización de medios electrónicos de entretenimiento y comunicación con la actividad física y estado nutricional de los adolescentes. [Disertación grado]. Pontificia Universidad Católica del Ecuador, Quito, Ecuador. Disponible en http://repositorio.puce.edu.ec/handle/22000/7512

Solis, M. (2014). Estado Nutricional, Patrones Alimentarios y Sedentarismo en Niños de Edad Escolar en Áreas Urbanas y Rurales de la Provincia Oropeza en ChuquisacaBolivia, 2011. AdAstra, 5(1), 8-12. Disponible en http://revistas.usfx.bo/index.php/ adastra

SPSS IBM (versión 22.0). Statistical Package for the Social Sciences. [Programa estadistico informático]. Armonk: IBM. Disponible en https://www.ibm.com/support/pages/ spss-statistics-220-available-download

TANITA. (TBF-300 GS-TBF MA). Body Composition Analyzer Goal Setter. [Equipo de medición]. Tokio: TANITA.

Zabaleta, S. (2012). ¿De qué manera el uso de aparatos tecnológicos afecta a niños pequeños? Revista KubernÉtica. Recuperado de https://www.santiagokoval.com/documentos/articulos-academicos/efectos-del-uso-de-dispositivos-tecnologicos-en-ninos-pequenos.pdf

Hendrick Márquez-Luengo: Licenciado en Nutrición y Dietética. Magister Scentiarium en Nutrición Comunitaria de la Universidad del Zulia (Venezuela). Doctorante del Programa de Ciencias de la Salud de la Universidad del Zulia (Venezuela).

Darío Acosta: Licenciado en Nutrición y Dietética. Universidad del Zulia (Venezuela).

Jorge Luis Chourio: Licenciado en Nutrición y Dietética. Universidad del Zulia (Venezuela).

José Reverón: Licenciado en Nutrición y Dietética. Universidad del Zulia (Venezuela). 